\title{
Student Innovation Process from Self-architecting Point of View
}

\author{
Mirtaleb Hosseini Kharvanagh ${ }^{1}$ \\ ${ }^{1}$ Department of Architecture, Islamic Azad University, Research and Science Branch, Zanjan, Iran \\ Correspondence: Mirtaleb Hosseini Kharvanagh, founder and the superior manager of the Open Mind \\ Architecture, the Student in Architecture Engineering in Higher Education, Islamic Azad University, Research \\ and Science, Zanjan Unit, Iran. E-mail: architect.mth@gmail.com
}

\author{
Received: February 29, 2016 Accepted: March 10, 2016 Online Published: April 19, 2016 \\ doi:10.5539/ass.v12n5p195 URL: http://dx.doi.org/10.5539/ass.v12n5p195
}

\begin{abstract}
Love to bubble the innovative creativity within oneself and increase the capacity of creativity capacity in human nature show the most striking difference between him and the other creations and will never be the end for this aim because man has always been following the attainment of immortality and is ahead of its era. The innovative creativity capacity of the mind is the most powerful incentives to form the processes that human could have been successful to represent it till today because human did a lot of searches and endeavors to access the eternity and being vanguard against the time over the time so as to find the solution to this cycle but he couldn't be successful in this field.

Lack of access to the innovative internal sources is because the man should at the first step be aware of philosophy, ignorance and life and critic them without the slightest consideration so that by attending self architect be aware of the value and the ability of the mind and by depending on own ingenuity move toward the creation of the new theoretical to outshine the time and lead up to the millennium of those won be and the powerful presence and immortality would be realized that it's output would be entering into the ultramodern mind cycle. The innovation means creating and providing new vents of the theoretical with the content of vanguard against the time. The process of entering to this cycle is so that the student must have believed his ignorance to be able to pass through his mental illness and feels the thirst of knowledge. Now he finds that having the eyes for viewing the wisdom and finding the ingenuity should pass via for main cycle to attend the knowledge and innovation through the sight of his architect (creator). Failure to pass the mentioned process expresses the shuck document with empty reason ownership. Realizing the process of forming own diagram and innovative design in this article has been delivered to the reader as discover page.
\end{abstract}

Keywords: self-architect's view, innovation, ultramodern mind, finding the ingenuity

\section{Introduction}

\subsection{Introducing the Problem}

Today, innovations in advanced societies has valuable place and has a very important determinant but this attitude in many nations towards innovation cycle does not pass a growing trend because educational system cycle in these communities is so that it wasn't defined for new creativity and ignoring it will lead to the formation of the least creative student as the philosophy of the students in the creation or discovery of new vents is of the theoretical but this process according to the existed realities cover a very slow and unsuitable movement in today's world. The reasons for the lack of innovation in students:

1- Lack of self-esteem and actual self confidence.

2- The fear of relying on internal capabilities for non passing through ignorance.

3- Searching out innovative creations in other's work.

4- Relying on the empty intelligence and lack of accessing the ingenuity.

5- Lack of enthusiasm on spontaneous research to explore the new vents

6- Coping the creations and annihilating the boiling inner creativity.

7- lack of understanding, companionship and conversation with his architect.

8- The role of the empty education system in the genesis of shells- oriented minds. 
9- The lack of enough protection of the innovative aspects of the research.

10- Representing the books without leading the time.

11- Lack of passing the teachers through training cycle rhetorical approach to train creative students.

12- Lack of a strong relationship between the three main sides meaning education system, students and industry.

13- Getting value the shell document to access the false social position.

\subsection{Methodology}

Innovation procedure in the article should be so that its creator could represent the new vent of the leading time idea which is born from the opening mind and observers eye so as to create such an eager and motivation in the mind of students so that every time he wants to scrape it, he creates a beautiful sculpture and new sight meaning springing the creativity in him should be a discover type and new creative to be able to introduce the new vents for research.

Exploratory type is of the best and the most creative methodology because in this method, the researcher must have an open mind and a discerning eye leading the time to be able to see the core of the theoretical content and observe and criticize express the duration of its use or their putrefaction and need to the new creativity.

Creating a new theoretical depends on the student who realizes the value and potential of such an innovative approach to enable self-discovery and creativity towards new creativity and move with a positive impact on self-esteem and self-confidence and believe the responsibility on the neck and believe in his own ability and ingenuity in the creation of new theories leading time to show them.

Exploratory research is one of the most amazing reasons of creating ultramodern minds to create new theoretical because it leads to the existence of his ability in new creativity and being affected from other works and show the new impetus for new researches.

\section{Theoretical}

\subsection{The Main Cycles of the New Creativities}

The new and pure creativity process should have such a structure that it could guide the student to discover the new vents in order not to rely on the other's mind contents and have dare to accept his ignorance and enters to the main four cycles meaning 1) Temperament: the element making up the one's nature. 2) Inside Nature: being aware of the structure formation process by itself that would be extremely important and valuable. 3) Thoughtfulness: observing the extract of the content clearly and attributes of words. 4) Self-fledged: awareness of the nature formation process and infra structures.

The above cycle causes the student to understand the language of self friendship and this process leads to the formation of companionship and conversation with the self architect himself so he learns that to release the innovation from the inside he should be aware of ingenuity and create thirst in him and for releasing the new innovation he close his mouth and ride on his thought and take the others away from his thoughts in order to record the new vents of theoretical and represent them.

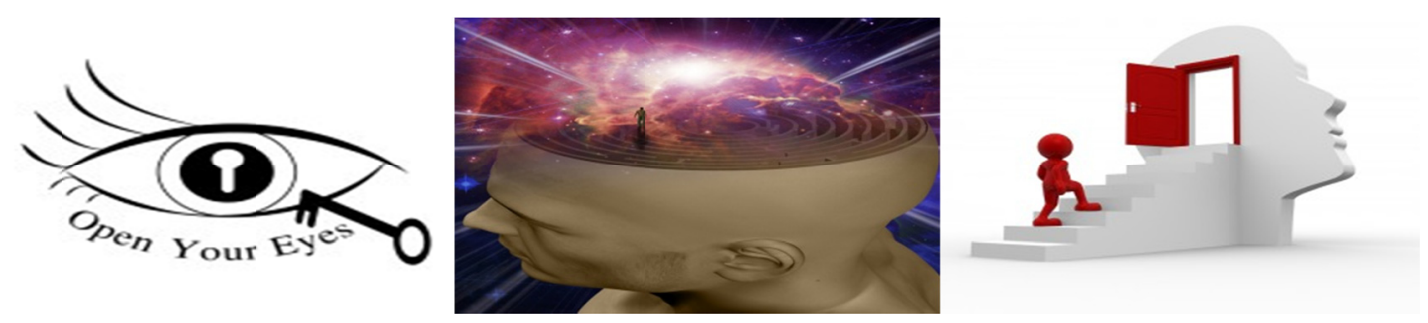

Figure 1. The pictures of self architect and the mind observer eye

This process resulted in the birth of a new leading time theorist who can investigate the other's work without being affected. So, the result of an innovative educational system is that enters the student into the open mind cycle to be able to create articles and books that never enters the decay process and by passing the time we find the new vents for research because the end of the education is creating leading time innovation.

The students should have an understanding of the cycle of innovation, ideas and creativity in order to obtain the 
cycle of the mind and have close relation with self architect so move to the new plan with leading time content.

The process of innovation is such that it should move via the mentioned cycles to be able to understand the life philosophy and challenge the existed theoretical foundations and figure out the creation of new vents and show the mental abilities. The structure of forming this process, according to our source, will be so that when enter to the observer eye, he will understand the philosophy of university education and move toward new thoughts and leading time in the view point of his creator.

The student will understand this subject that without awareness of the structure of his nature he will not attend the nature of the formation process because temperament is the nearest vent for knowing the inner nature and lack of passing this process is ignoring the ingenuity of the architect and cause to choose the course based on the social situations and he will not aware of his fate so entering the self-fledged will be destroyed.

\subsection{Ingenuity and the Process of Finding It}

Own ingenuity is the most powerful immortal asset in every man that the process of knowing it depends on passing the students through the mentioned cycles to be able to start the innovation from the inside. Being aware of ingenuity and apply it cause that we get the most wonderful inner peace by releasing the new creativity. Because this property show the leading time observers eye and create enough motivation to new vents and force him to think deeply about the past start the future and will be able to see the subjects clearly.

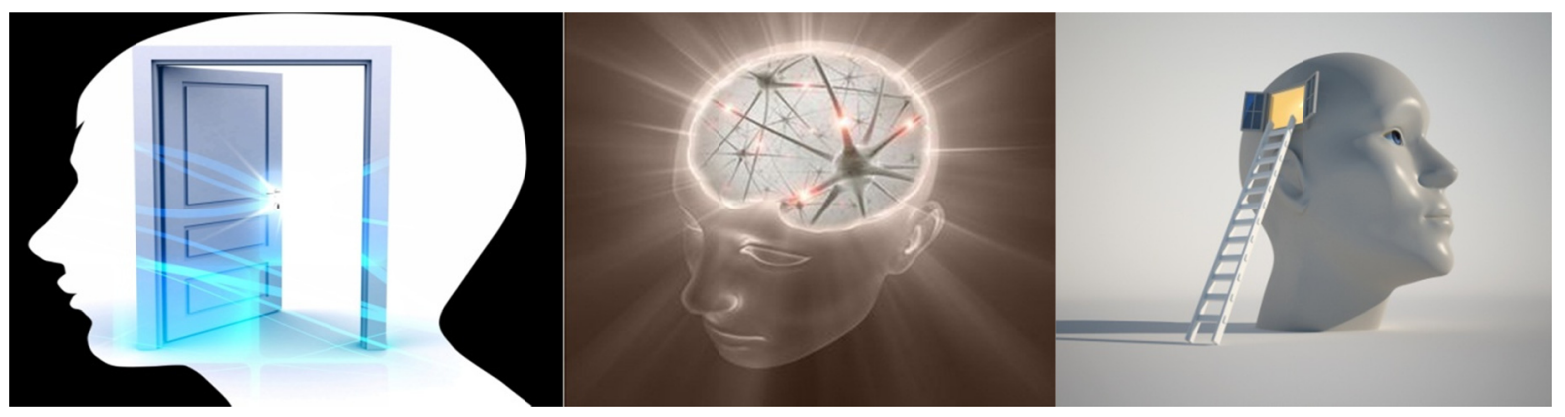

Figure 2. The pictures of open mind

Now the student has gained the knowledge on the origin of the formation process and can create fundamental changes in it. The above process suggests that knowledge of the nature and structure of ingenuity, ideas and creativity have higher importance and value. Because it created the open mind and creative new thinking and it will have creation of new knowledge after itself.

\subsection{The Empty Consciousness and the Creation of Shell-Oriented Mind}

The empty consciousness is resulted from own ignorance and is the reason of creating the shell -oriented mind. Non-Proliferation architect at self-sustaining vent caused the formation of empty consciousness and shell-oriented mind and as a result he couldn't be able to win the nature of the innovation structure and represent a strong reply to the questions of the masters and the society. In the view point of them, he only entered the university to get a shell document and find a false social position.
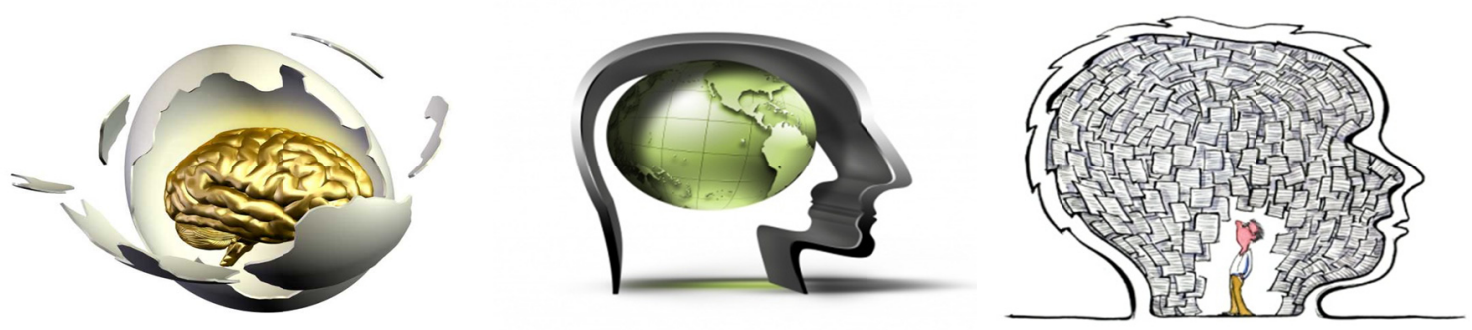

Figure 3. The pictures of the mind with shelf 
This process makes him a social person with empty consciousness who is only a leech on the other's mind and finally his ability would be creating a meaningless shell. Because the empty consciousness creates an empty mind that its base is decay words. The shell-oriented mind causes that the ingenuity will be abortive in the student and makes him as a moving scarecrow having no innovation which is not able to rely on his architect.

There is a lot of suffering and hardship in his way that cause him to take steps towards self away and caused that he find the solution in other's minds and this process create a thought that other's work is the best in his empty consciousness and constantly he moves toward them and kill the time and the life in these useless thoughts while the only way to live with own architect is to embrace your own ingenuity.

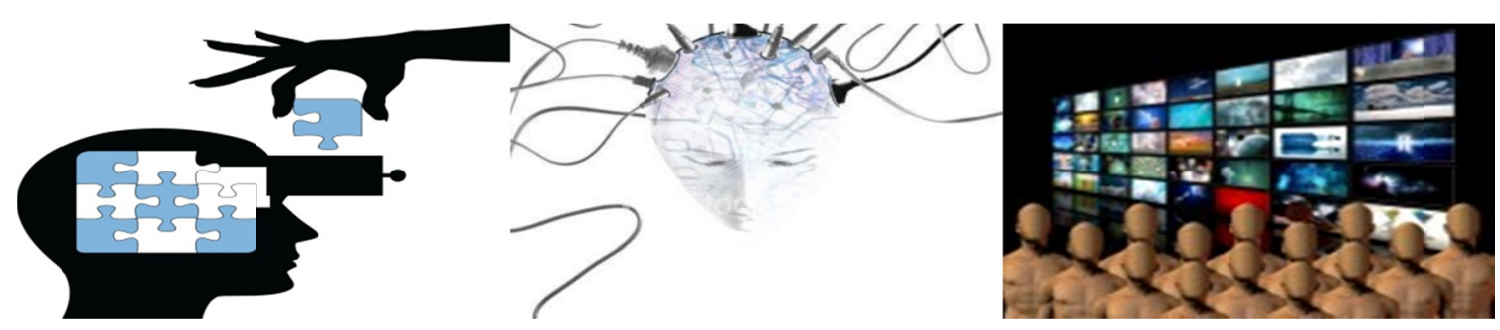

Figure 4 . The pictures of the mind venom

The empty consciousness has destroyed the new creativity and cause not to think about mental poisoning that prevents to make process of vomiting and it will follow a vortex of mental slavery. This process provide a base for closed mind and create a base which is decay from the inside for the student and omit the leading time observer ability and also creates a shell knowledge that destroys own ingenuity architect.

\subsection{Wisdom Role in Innovation}

The wisdom is the observers which with we can see the final of the new theoretical creativity before entering the applied cycle clearly and challenge and criticize its contents. Wisdom has constructive role in the process of innovation is when the students obtain a thirst for knowledge and love of self discovery to lead to the emergence of open mind and to observer eye.

As long as the student misses the philosophy of ignorance he can't gain the value of innovation.

Failure to pass the above cycle causes not to see a various vents of the wisdom. Wisdom creates the ability to see the end and before the beginning even leading time and the value of their content value. This ability cause to form a powerful tool called preventive intelligence. Preventive intelligence is a factor that makes students to distinguish the difference between ignorance and wisdom and move to the new different thinking and in this way he will be familiar to the different points of view and understand the ultramodern mind.

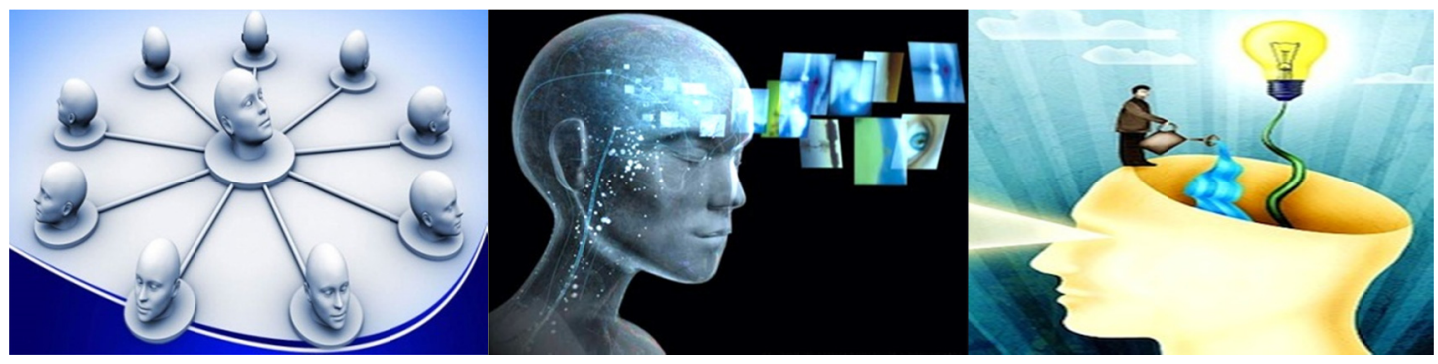

Figure 5. The pictures of ultramodern mind

The process of the rational dialogue from the open mind vents with his genius architect makes the student create the new vents of leading time theoretical and know their application and the process of forming their content and their structure.

Taking value by new creativity will prepare him for knowing his mental abilities to follow the difficulties of this way and move to the true content of the logic, intelligence and new thought to prevent the ineffective life. Now he can by relying on the genius of the architect make the ahead time clear and can have the ability to observe the 
new world and the content of it and experience the joy of life.

If the structure of the leading time innovation cycle is applied in the education system it will be the messenger of forming the most powerful tool for fostering open minds and observing eyes. This process shows that the number of innovative student with extraordinary speed will increase. Application of this theory requires a keen and armed eye because they would only create antidote to mental poisoning of students and the presence of this great master in a position to achieve an open mind and creative vent will accelerate the leading time theoretical.

Now the community is able to feel the progress and the joy of movement and by relying on the leading students can have new thoughts and logic. These amazing tools enable the students before creating a the new theoretical to consider a place for his wisdom and by applying that, create new nature and structure because the ability of the mind for criticizing is infinite that there is not any power that could be compared with it. So, the wisdom has a special place in innovative and ignoring it create empty bases and make the process of the educational system ill and such a society is not able to fight with breakers structures. Knowledge of the secret wisdom is of the most surprising part of the legacy for all ages because achieving this important and valuable cycle will be possible only through the nation who passionate love of wisdom and nurturing minds and put his life on the movement towards the creation of fundamental knowledge.

\section{Findings}

As mentioned in the theoretical papers lack of the application the above process prevents the inner creativity because the process of innovation in the educational system should pass via the cycles so that the student be armed with open mind and wisdom eye and move to the ultramodern mind and keep the motivation for sustainable development in the society.

If the educational system is rotten from the closed minds and decay thoughts it will be the messenger of decay thoughts because this movement in such a based remove the ability to create the new vents because of the accumulation of others' work in the mind.
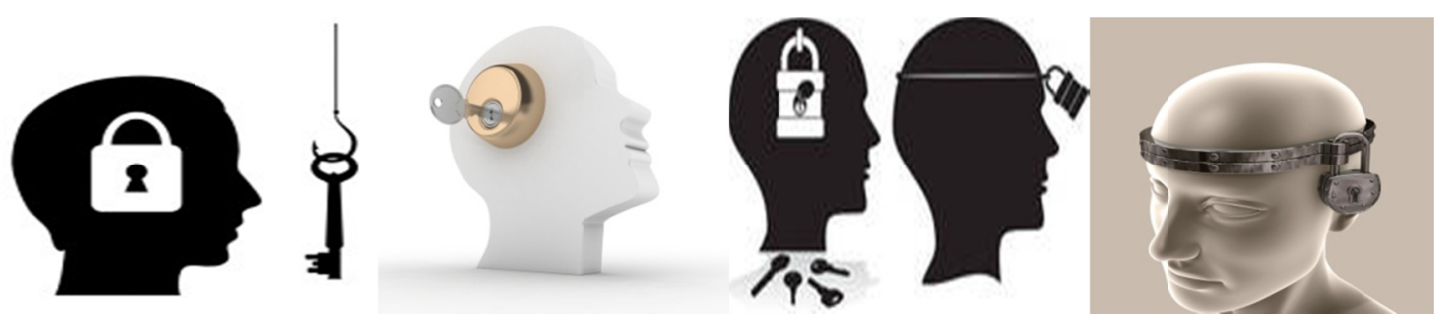

Figure 6. The pictures of closed mind

Such a cycle can't guide the student to understanding, relying on the genius of own architect. Because destroy the accessing to the creative source of new ideas and the conditions of copying the works of others, which destructive heritage of the educational system lacks this infrastructure.

If the structure for any reason ignores the attitude of innovative student via the vent of his architect view will be cause of destruction within the society and as a result will not be able to pass the competitors in his life.

The purpose of the formation process of the educational system is that students can discover new vents from theoretical expressions and result in innovation, progress, dynamism and happiness because it is his open minds and observer eyes which is the best vent for forming the new process.

\section{Summary and Conclusion}

The quiet mind doesn't have a place for live in the philosophy of college education. If the process of raising creative student in the educational system is ignored, it will destroy ingenuity and this happening will destroy the ability to enter the new creative cycle. Because the min point is that we should destroy the belief of creating idol in false society to the inner creative spring would be appeared.

Ultramodern mind should have such a privileged position in the society that the student could provide a factor for development by relying on ingenuity because he can completely change the culture and economy and it is possible by relying on the open and creative mind. The student is nearest and full of the contents of the thought vent of a society and its purpose is accessing the open mind and wisdom eye, creating amazing heritage for all the ages and accomplish with his architect. So if the society fails to understand the philosophy of his architect, 
his elites will be in the arms of its rivals.

\section{Reference}

Hosseini Kharvanagh, M. (2014). Companionship glory with self architect. Tehran, Bishe Publication.

\section{Copyrights}

Copyright for this article is retained by the author(s), with first publication rights granted to the journal.

This is an open-access article distributed under the terms and conditions of the Creative Commons Attribution license (http://creativecommons.org/licenses/by/3.0/). 